NOTICE: this is the author's version of a work that was accepted for publication in Preventive Medicine. Changes resulting from the publishing process, such as peer review, editing, corrections, structural formatting, and other quality control mechanisms may not be reflected in this document. Changes may have been made to this work since it was submitted for publication. A definitive version was subsequently published in Preventive Medicine, Vol. 54, No. 6 (2012). DOI: 10.1016/j.ypmed.2012.03.015 
Effects of a Physical Activity and Nutrition Program for Seniors on Body Mass

Index and Waist-to-Hip Ratio: A randomised controlled trial

Linda Burke ${ }^{1,2}$, Andy H. Lee ${ }^{1,2}$, Maria Pasalich ${ }^{1}$, Jonine Jancey ${ }^{1,2}$, Deborah Kerr ${ }^{1}$, Peter

Howat $^{1,2}$

${ }^{1}$ School of Public Health

${ }^{2}$ Centre for Behavioural Research in Cancer Control

Curtin University

GPO Box U 1987

Perth, Western Australia, 6845

Corresponding Author

L.Burke@curtin.edu.au

Phone: +61-8-92664535

Fax: +61-8-92662958

Co-authors

Andy.Lee@curtin.edu.au

Maria.Pasalich@curtin.edu.au

J. Jancey@ curtin.edu.au

D. Kerr@curtin.edu.au

P.Howat@curtin.edu.au 


\title{
Effects of a physical activity and nutrition program for seniors on body mass index and waist-to-hip ratio: A randomised controlled trial
}

\author{
ABSTRACT \\ Objective: To investigate whether a home-based program, physical activity and nutrition for \\ seniors (PANS), made positive changes to central obesity, measured by body mass index \\ (BMI) and waist-to-hip ratio (WHR).
}

Methods: A 6-month randomised controlled trial was conducted targeting overweight and sedentary older adults aged 60 to 70 years residing in low to medium socio-economic suburbs within metropolitan Perth. Intervention participants $(n=248)$ received mailed materials and telephone/email support to improve nutrition and physical activity levels. Controls $(n=230)$ received small incentives to complete baseline and post-intervention questionnaires. Both groups reported anthropometric measures following specific written instructions. Generalised estimating equation models were used to assess repeated outcomes of BMI and WHR over both time points.

Results: 176 intervention and 199 controls (response rate 78.5\%) with complete data were available for analysis. After controlling for demographic and other confounding factors, the intervention group demonstrated a small (0.02) but significant reduction in WHR (p = 0.03) compared to controls, no apparent change in BMI was evident for both groups. The 0.02 reduction in mean WHR corresponded to a $2.11 \mathrm{~cm}$ decrease in waist circumference for a typical hip circumference.

Conclusion: PANS appears to improve the WHR of participants. Changes in BMI might require a longer term intervention to take effect, and/or a follow-up study to confirm its sustainability.

Trial Registration: anzctr.org.au Identifier: ACTRN12609000735257

Key words: goal setting; intervention; nutrition; physical activity 


\section{INTRODUCTION}

Obesity has reached epidemic proportions globally, with more than one billion adults overweight and at least 300 million of them clinically obese (World Health Organization, 2011). In Australia, the ageing population is heavier than they were a generation ago, with more than 79\% of older Australians (aged 65-74 years) now considered as obese or overweight (Australian Bureau of Statistics, 2009). Obesity is a major contributor to the global burden of chronic disease and disability (World Health Organization, 2011). It is also a costly public health problem. The nationwide annual cost of obesity in Australia is estimated to be $\$ 21$ billion (Physical Activity Taskforce, 2007). Due to the ageing population, sound interventions capable of making a positive change to the health status of older adults are needed to curb the obesity epidemic. However, to date there is evidence that communitybased interventions have had limited success in gaining substantial or lasting benefits (Walls et al., 2011).

Although the main causes of obesity are increased consumption of energy-dense foods high in saturated fats and sugars, and reduced physical activity (World Health Organization, 2011), many intervention programs for seniors have focused either solely on physical activity (Cox, 2008; Greaney et al., 2008; Jancey et al., 2008; Wilcox et al., 2008) or diet alone (Greene et al., 2008; Johnson et al., 2004). Recently, a few programs have considered both aspects with some promising results (Morey et al., 2009; Villareal et al., 2011). Among them, the physical activity and nutrition for seniors (PANS) program attempted to control overweight and obesity by improving both physical activity and dietary behaviour (reduce fat, increase fibre, fruit and vegetable intake). It was a low cost and accessible home-based intervention developed specifically for insufficiently active older adults aged 60 to 70 years (Burke et al., 2010) . At the conclusion of the program, participants were found to increase their walking time and strength activities while reducing their sitting time and fat intake.

Anthropometric measurements are typically used to assess the effectiveness of weight management and obesity control trials (Goodpaster et al., 2010). Apart from body mass index (BMI), waist-to-hip ratio (WHR) has been shown to be a good indicator of cardiovascular disease (Esmaillzadeh et al., 2004; Welborn and Dhaliwal, 2007), trunk fat mass (Taylor et al., 2000) and all-cause mortality (Welborn and Dhaliwal, 2007). In the absence of accurate clinical measurements which are preferable, self-reported data are often taken as the proxy, 
especially in large community-based trials to reduce cost and attrition rates (Dhaliwal et al., 2010). Recent research has confirmed the effectiveness of such self-reported anthropometric measures (Burton et al., 2010; Dhaliwal et al., 2010; Fillenbaum et al., 2010; Stommel and Schoenborn, 2009).

This study aims to determine whether the PANS intervention has made a positive impact on the outcome measures of self-reported BMI and WHR. The findings have important implications on the control and prevention of central obesity for older adults.

\section{METHODS}

\section{Study Design and Procedure}

PANS was a six-month two-arm randomised controlled trial delivered by mailed materials and telephone calls. It was developed as a home-based intervention with the intention to accommodate insufficiently active seniors who could semi-tailor the program to suit their own pace and needs (Burke et al., 2010). The project protocol was approved by the Human Research Ethics Committee of the researchers' institution and written consent was obtained from all participants.

In 2010 a total of 478 participants residing in low to medium socio-economic suburbs within metropolitan Perth were recruited from the Australian Federal Electoral Roll and randomly assigned to the intervention $(n=248)$ and control $(n=230)$ groups using a table of random numbers. More participants were allocated to the intervention group due to its expected higher attrition rate. Older adults aged between 60 and 70 years satisfied the selection criteria if they (a) were not on any special diet and (b) were "insufficiently active", i.e. participated in less than 30 minutes of moderate activity on at least 5 days per week (Brown et al., 2008), but (c) were considered "healthy" to the extent that participation in a low-stress physical activity program would not place them at risk. Figure 1 shows the flow chart of the recruitment procedure and corresponding sample sizes; details of which had been reported previously (Burke et al., 2010).

\section{Intervention}

The PANS intervention consisted of a specially designed booklet containing information on dietary guidelines, recommended physical activity levels and encouraged goal setting. The 
booklet was supplemented by additional written materials including an exercise chart, calendar and bi-monthly newsletters. The participants were also given a pedometer to keep track of their daily steps and a resistance band to perform strength exercises suggested in the booklet. Strength exercises were intended to reduce fat mass and help increase muscle mass (Banz et al., 2003; DeNysschen et al., 2009). Moreover, group guides provided telephone and email support, as well as the coordination of non-compulsory group meetings. The PANS participants could semi-tailor the program to suit their own pace and need, and participated as much or as little as they wanted without restriction over the six-month period. Further information on the intervention can be found elsewhere (Burke et al., 2010). The program resources were subsequently evaluated using a feedback sheet. Controls received baseline and post-intervention questionnaires at the same time as the PANS participants and were given a small incentive upon completion and return of the postal questionnaires.

\section{Anthropometric Measures}

In addition to demographic and lifestyle characteristics, physical activity and dietary behaviours, the self-completion questionnaires solicited information on height, weight, waist and hip girth measurements at baseline and at six months post intervention. All subjects were provided with a tape measure and clearly written instructions on how to accurately measure themselves at both time points. They were reminded not to wear shoes when measuring height and weight. The instructions explained how to measure waist circumference "halfway between the lowest rib and top of hipbone, roughly in line with the belly button", and hip circumference "at the point where the buttocks protrude the most". Subjects were requested to "breathe out normally", then "measure directly against their skin and make sure the tape was snug and not twisted, without compressing the skin", before recording to the nearest 0.5 inch or cm (Commonwealth of Australia, 2009).

\section{Statistical Analysis}

Descriptive statistics were first applied to summarise the baseline demographic profile and lifestyle characteristics of the sample. The continuous outcomes of BMI and WHR were then compared between intervention and control groups across the two time points using independent samples and paired t-tests. To accommodate the inherent correlation of observations taken from the same individual, generalised estimating equation (GEE) models with exchangeable correlation structure were fitted to assess the two repeated measures over 
time, while accounting for the effects of potential confounding factors. An intention-to-treat analysis was also performed to assess the sensitivity of the analysis. All statistical analyses were undertaken in the SPSS package version 18.

\section{RESULTS}

Of the total 478 recruited seniors who completed the baseline questionnaire, 176 intervention participants and 199 controls with complete data were available for analysis, giving a final response rate of $78.5 \%$. Table 1 presents the characteristics of the completers at baseline. The intervention and control groups were similar in terms of demographic and lifestyle variables. Overall, the mean age was 66 years, about half were male, the great majority of them had a partner and experienced common health conditions prevalent among older adults. Just less than half the seniors received tertiary education while over $40 \%$ continued working. No differences in alcohol drinking and smoking status were found between the intervention participants and controls.

Table 2 compares the BMI and WHR outcomes between the intervention and control groups across the two time points. The mean BMI measures at six months were similar to those at baseline for both groups. However, a significant improvement in mean WHR from baseline to post- program was evident among the intervention participants but not the controls, even though the reduction of 0.02 appeared small in magnitude. This 0.02 reduction in mean WHR corresponded to a $2.11 \mathrm{~cm}$ decrease in waist circumference for a typical hip circumference among the intervention participants.

Results of the GEE analyses are given in Table 3. After controlling for demographic and other confounding factors, the intervention group demonstrated a significant reduction in WHR ( $\mathrm{p}=$ 0.03) relative to the controls, but no change in BMI was apparent for both groups through the group xtime interaction term. In addition, WHR was positively associated with the male gender, whereas the presence of co-morbidity had more significant impact on the seniors' BMI than their WHR. The estimated correlations between the repeated observations were substantial which justified the fitting of GEE models.

An intention-to-treat analysis was next performed. For both intervention and control groups, no significant differences in baseline variables were found between completers and non- 
completers, so that the post-program BMI and WHR outcomes of the latter could be considered as missing at random (results omitted for brevity). With the inclusion of the noncompleters' baseline data, the corresponding GEE fits are shown in Table 4. The results were generally comparable with those in Table 3 , though the effect of the group $\times$ time interaction term became marginal $(\mathrm{p}=0.06)$ for WHR.

Process evaluation of the program resources provided the following feedback. The great majority ( $80 \%$ ) of PANS participants reported information given in the booklet was useful, easy to understand and suitable for their age group, which encouraged them to think about physical activity and nutrition behaviours. They believed the exercise chart was appropriate (74\%) and used the resistance band provided to practise the suggested stretch and strength exercises (63\%). Over $90 \%$ of participants said they used the pedometer. Moreover, many participants (64\%) perceived that the provision of additional support through group guides could facilitate individuals to set and achieve personal physical activity and nutrition goals.

\section{DISCUSSION}

This study investigated the effectiveness of the PANS intervention in terms of anthropometric end points. The moderate sample sizes provided sufficient statistical power for evaluation of the repeated measures (Burke et al., 2010). As expected, the attrition was higher among the intervention participants (29\%) than the control group (13.5\%), but the rates were comparable with similar physical activity trials in the literature (Cox, 2008; Jancey et al., 2008). Future studies should take account of barriers when designing programs for older adults, and may consider using computer-assisted telephone interviewing (Wilson et al., 2001) instead of selfcompletion postal questionnaire to improve the response rate. Moreover, process evaluation of PANS revealed that the program strategies and resources were relevant to the target group, assisting participants to increase their level of physical activity and improve dietary intake.

According to Dhaliwal and Welborn (2009), the difference between two successive measurements in a subject will be considered significant in clinical practice if the value is greater than the measurement error, which is 0.02 for WHR for both males and females. Therefore, the mean WHR reduction of 0.02 observed in the intervention group would be regarded as clinically relevant. Despite being home-based, the PANS intervention was successful in motivating participants to engage in walking and strength exercises while 
reducing their sitting time and fat intake (Lee et al., 2011). It is likely that these positive behavioural changes contributed to their significant decrease in WHR over the six-month period. As changes in central obesity can be effectively measured via WHR (Welborn and Dhaliwal, 2007), the observed improvement in WHR among the PANS participants have important consequences for implementing obesity control and management for older adults in the clinical setting.

Although the recommended strength exercises using the resistance band might increase the build-up of muscle mass (Banz et al., 2003; DeNysschen et al., 2009), the reduction in BMI among the intervention participants was not statistically significant. A longer time frame for the intervention program is probably required to demonstrate substantial changes in BMI. A follow-up study to collect anthropometric measurements is also recommended to confirm the sustainability of the PANS intervention beyond six months.

Two pertinent factors were found to be associated with BMI and WHR. In this sample, males had significantly higher WHR than their female counterparts, whereas the presence of some common health conditions (heart disease, stroke, diabetes, cancer, osteoporosis, arthritis, high blood pressure, high cholesterol and constipation) impacted positively on the anthropometric outcomes, especially BMI. These effects were expected and consistent with the literature; WHR tends to be higher in men than women (Ferrara et al., 2008; Lee et al., 2005; Wu et al., 2007), and the existence of chronic health conditions have been associated with elevated BMI (Must et al., 1999).

A potential limitation of the study concerned the self-reporting of anthropometric measures, though the inherent inaccuracies were expected to be similar between the intervention and control groups. All subjects were provided with a tape measure and clearly written instructions on how to take measurements at both time points. Self-reported data have been considered as valid proxies to reduce cost and attrition rates in large scale community trials, and should be sufficiently reliable for monitoring changes over time (Burton et al., 2010; Dhaliwal et al., 2010; Fillenbaum et al., 2010; Stommel and Schoenborn, 2009), which formed the basis of our evaluation. Self-selection bias was minimised through randomisation, but participation was voluntary due to the home-based nature of the intervention. Therefore, reporting bias might still pose a threat to the validity of the findings. Moreover, residual 
confounding could not be eliminated even though demographic and other factors were adjusted for in the GEE regression analyses. As alluded above, the duration of the PANS intervention might not be of sufficient length to reflect significant changes in BMI. A longer, sustainable program should be considered in the future, but it might increase the likelihood of further attrition among older adults. 


\section{Conflict of interest}

Nothing declared for all authors.

\section{Acknowledgements}

The authors are grateful to the seniors who participated in this project. The study was funded by a research project grant from the Australian National Health and Medical Research Council (project number 533501) and the Centre for Behavioural Research in Cancer Control. The funding sources had no involvement in the study design, data collection, analysis or writing of papers including decisions in regard to submission of papers. The project protocol was approved by the Human Research Ethics Committee of Curtin University (approval number HR 186/2008). 


\section{References}

Australian Bureau of Statistics, 2009. National Health Survey: Summary of Results, 20072008, Canberra, A. C. T.

Banz, W.J., Maher, M.A., Thompson, W.G., Bassett, D.R., Moore, W., Ashraf, M., Keefer, D.J., Zemel, M.B., 2003. Effects of Resistance versus Aerobic Training on Coronary Artery Disease Risk Factors. Exp Biol Med 228:434-40.

Brown, W.J., Moorhead, G.E., Marshall, A.L., 2008. Choose health:Be Active:A physical activity guide for older Australians, 3rd ed. Commonwealth of Australia and repatriation Commission, Canberra.

Burke, L., Jancey, J., Howat, P., Lee, A., Kerr, D., Shilton, T., Hills, A., Anderson, A.S., 2010. Physical activity and nutrition program for seniors (PANS): protocol of a randomized controlled trial. BMC Public Health 10:751.

Burton, N., Brown, W., Dobson, A., 2010. Accuracy of body mass index estimated from selfreported height and weight in mid-aged Australian women. Aust N Z J Public Health 34:4.

Commonwealth of Australia, 2009. Measureup Campaign. Department of Health and Ageing [online], available:

http://www.measureup.gov.au/internet/abhi/publishing.nsf/Content/factsheet-waistmeasurement [accessed October 2009].

Cox, K.L., Burke, V, Beilin, L.J, Derbyshire, A.J, Grove, J.R, Blanksby, B. A, Puddey, I.B, 2008. Short and long-term adherence to swimming and walking programs in older women-the Sedentary Women Exercise Adherence Trial (SWEAT 2). Prev Med 46:51117.

DeNysschen, C., Burton, H., Horvath, P., Leddy, J., Browne, R., 2009. Resistance training with soy vs whey protein supplements in hyperlipidemic males. J Int Soc Sports Nutr $6: 8$.

Dhaliwal, S., Howat, P., Bejoy, T., Welborn, T., 2010. Self-reported weight and height for evaluating community obesity studies. Am J Health Behav. 34:489-99.

Dhaliwal, S.S., Welborn, T.A., 2009. Measurement error and ethnic comparisons of measures of abdominal obesity. Prev Med 49:148-52.

Esmaillzadeh, A., Mirmiran, P., Azizi, F., 2004. Waist-to-hip ratio is a better screening measure for cardiovascular risk factors than other anthropometric indicators in Tehranian adult men. Int J Obes Relat Metab Disord 28:1325-32. 
Ferrara, C., Goldberg, A., Nicklas, B., Sorkin, J., Ryan, A., 2008. Sex differences in insulin action and body fat distribution in overweight and obese middle-aged and older men and women. Appl Physiol Nutr Metab 33:7.

Fillenbaum, G.G., Kuchibhatla, M.N., Whitson, H.E., Batch, B.C., Svetkey, L.P., Pieper, C.F., Kraus, W.E., Cohen, H.J., Blazer, D.G., 2010. Accuracy of Self-reported Height and Weight in a Community-Based Sample of Older African Americans and Whites. J Gerontol A Biol Sci Med Sci s 65A:1123-29.

Goodpaster, B.H., DeLany, J.P., Otto, A.D., Kuller, L., Vockley, J., South-Paul, J.E., Thomas, S.B., Brown, J., McTigue, K., et al., 2010. Effects of Diet and Physical Activity Interventions on Weight Loss and Cardiometabolic Risk Factors in Severely Obese Adults. JAMA 304:1795-802.

Greaney, M.L., Riebe, D., Ewing Garber, C., Rossi, J.S., Lees, F.D., Burbank, P.A., Nigg, C.R., Ferrone, C.L., Clark, P.G., 2008. Long-term effects of a stage-based intervention for changing exercise intentions and behavior in older adults. Gerontologist 48:358.

Greene, G.W., Fey-Yensan, N., Padula, C., Rossi, S.R., Rossi, J.S., Clark, P.G., 2008. Change in fruit and vegetable intake over 24 months in older adults: results of the SENIOR project intervention. Gerontologist 48:378.

Jancey, J., Clarke, A., Howat, P., Lee, A., Shilton, T., Fisher, J., 2008. A physical activity program to mobilize older people: A practical and sustainable approach. Gerontologist $48: 251-57$.

Johnson, D., Beaudoin, S., Smith, L., Beresford, S., LoGerfo, J., 2004. Increasing fruit and vegetable intake in homebound elders: the Seattle Senior Farmers' Market Nutrition Pilot Program. Preventing Chronic Disease [serial online] date cited 28-11-11 Available from URL: http://www.cdc.gov/pcd/issues/2004/jan/03_00010a.htm.

Lee, A., Jancey, J., Howat, P., Burke, L., Kerr, D., Shilton, T., 2011. Effectiveness of a homebased physical activity and nutrition pilot program for seniors. J Obes 2011:8.

Lee, C.C., Glickman, S.G., Dengel, D.R., Brown, M.D., Supiano, M.A., 2005. Abdominal Adiposity Assessed by Dual Energy X-Ray Absorptiometry Provides a Sex-Independent Predictor of Insulin Sensitivity in Older Adults. J Gerontol A Biol Sci Med Sci 60:87277.

Morey, M., Snyder, D., Sloane, R., Cohen, H., Peterson, B., Hartman, T., Miller, P., Mitchell, D., Demark-Wahnefried, W., 2009. Effects of home-based diet and exercise on functional outcomes among older, overweight long-term cancer survivors. JAMA 301:1883-91. 
Must, A., Spadano, J., Coakley, E.H., Field, A.E., Colditz, G., Dietz, W.H., 1999. The

Disease Burden Associated With Overweight and Obesity. JAMA 282:1523-29.

Physical Activity Taskforce, 2007. Premier's Physical Activity Taskforce: strategic plan 20072011. Government of Western Australia.

Stommel, M., Schoenborn, C., 2009. Accuracy and usefulness of BMI measures based on self-reported weight and height: findings from the NHANES \& NHIS 2001-2006. BMC Public Health 9:421.

Taylor, R., Jones, I., Williams, S., Goulding, A., 2000. Evaluation of waist circumference, waist-to-hip ratio, and the conicity index as screening tools for high trunk fat mass, as measured by dual-energy X-ray absorptiometry, in children aged 3-19 y. Am J Clin Nutr $72: 6$.

Villareal, D.T., Chode, S., Parimi, N., Sinacore, D.R., Hilton, T., Armamento-Villareal, R., Napoli, N., Qualls, C., Shah, K., 2011. Weight Loss, Exercise, or Both and Physical Function in Obese Older Adults. N Engl J Med 364:1218-29.

Walls, H., Peeters, A., Proietto, J., McNeil, J., 2011. Public health campaigns and obesity - a critique. BMC Public Health 11:136.

Welborn, T.A., Dhaliwal, S.S., 2007. Preferred clinical measures of central obesity for predicting mortality. Eur J Clin Nutr 61:1373-79.

Wilcox, S., Dowda, M., Leviton, L.C., Bartlett-Prescott, J., Bazzarre, T., Campbell-Voytal, K., Carpenter, R.A., Castro, C.M., Dowdy, D., et al., 2008. Active for Life: Final Results from the Translation of Two Physical Activity Programs. Am J Prev Med 35:340-51.

Wilson, D., Taylor, A., Chittleborough, C., 2001. The Second Computer Assisted Telephone Interview (CATI) Forum: The state of play of CATI survey methods in Australia. Aust N Z J Public Health 25:272-74.

World Health Organization, 2011. Global Strategy on Diet, Physical Activity and Health. Obesity and Overweight. March 2011 ed. World Health Organization.

Wu, C.H., Heshka, S., Wang, J., Pierson, R.N., Jr., Heymsfield, S.B., Laferrere, B., Wang, Z., Albu, J.B., Pi-Sunyer, X., et al., 2007. Truncal fat in relation to total body fat: influences of age, sex, ethnicity and fatness. Int J Obes 31:1384-91. 